\title{
THE OBSERVATIONS OF 80-MIN OSCILLATIONS IN THE QUIESCENT PROMINENCES*
}

\author{
V. S. BASHKIRTSEV, N. I. KOBANOV, and G. P. MASHNICH \\ Siberian Institute of Terrestrial Magnetism, Ionosphere and Radio Wave Propagation, (SibIZMIR), \\ Irkutsk 33, P.O. Box 4, U.S.S.R.
}

\begin{abstract}
Oscillations of the line-of-sight velocities with periods $82^{\mathrm{m}} \cdot 2$ and $76^{\mathrm{m}} \cdot 7$ were detected in quiescent prominences, with coordinates $\varphi=-75^{\circ} \mathrm{W}$ and $\varphi=-18^{\circ} \mathrm{W}$, respectively.
\end{abstract}

At Sayan Observatory, Bashkirtsev and Mashnich have been successful in taking the first observations of line-of-sight velocity oscillations in two limb prominences with coordinates: (1) $\varphi=-75^{\circ} \mathrm{W}$ and (2) $\varphi=-18^{\circ} \mathrm{W}$ on 30 and 31 March, 1981.

Within the least years, the highly sensitive differential methods (Kalinyak and Vasilyeva, 1971; Kotov et al., 1978, 1982) have been extensively used for line-of-sight velocity measurements. We employed a differential technique designed for the study of local quasi-periodical motions of solar matter (Kobanov, 1983).

The principle underlying our method is that we measure the difference of Doppler shift of two line profiles from two areas on the Sun's surface using the same spectral line, in our case the $\mathrm{H} \beta$-line. The separation of the two measured areas, each having the size $5.0 \times 4 . " 4$ in the prominences was 4 " along the radius. The apparent height above limb at the site of measurement was respectively $\sim 1.5 \times 10^{4} \mathrm{~km}$ and $\sim 10^{4} \mathrm{~km}$ for prominences (1) and (2). As revealed by an inspection of the spectrum, the two prominences displayed no significant line-of-sight velocities or apparent changes in their profiles for the time of recording the oscillations. Therefore one can consider them as quiescent objects.

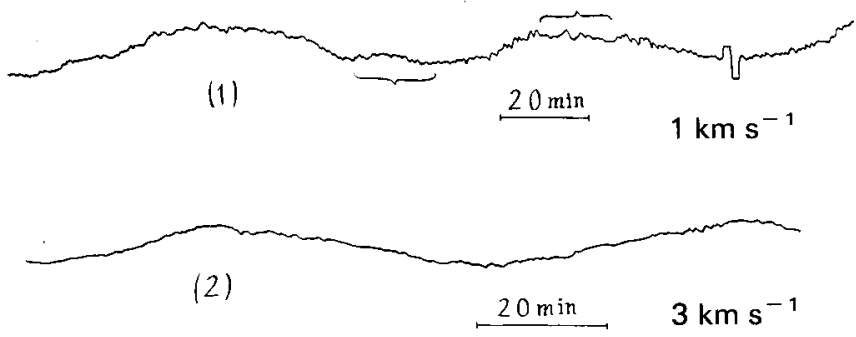

Fig. 1. Copies of records of prominence line-of-sight velocity oscillations: (1) $\varphi=-75^{\circ} \mathrm{W}, 30 \mathrm{March}$, $1981,02^{\mathrm{h}} 54^{\mathrm{m}} \leq \mathrm{UT} \leq 06^{\mathrm{h}} 00^{\mathrm{m}}$. Parts of the record marked by the braces have been obtained through cirrus clouds. The calibration procedure appears at the end of the record: (2) $\varphi=-18^{\circ} \mathrm{W}, 31 \mathrm{March}, 1981$, $02^{\mathrm{h}} 16^{\mathrm{m}} \leq \mathrm{UT} \leq 04^{\mathrm{h}} 10^{\mathrm{m}}$.

* Proceedings of the 66th IAU Colloquium: Problems in Solar and Stellar Oscillations, held at the Crimean Astrophysical Observatory, U.S.S.R., 1-5 September, 1981. 
We made measurements with a magnetograph, with one photomultiplier tube and automatic compensation of brightness fluctuations and information output on a strip-chart recorder (Lebedev et al., 1972). Prominence image on the spectrograph entrance slit was kept by a photoelectric guide to an accuracy of 1 arc sec.

The record copies of the line-of-sight prominence velocities are shown in Figure 1. The straight lines indicate time and amplitude scales. The registration length for prominence (1) is longer than two oscillation periods $\left(3^{\mathrm{h}} 06^{\mathrm{m}}\right)$, and for prominence (2), one period and a half $\left(1^{\mathrm{h}} 54^{\mathrm{m}}\right)$.

The reduction of the oscillation records was carried out using correloperiodogram analysis (Kopecký and Kuklin, 1971). The reduction used data series consisting of 840 and 520 points for prominences (1) and (2), respectively. One digitizing step is $12.5 \mathrm{~s}$.

The reduction results are shown in Figure 2, where $T$ is the period in seconds and $\rho$ the correlation coefficient that had been inferred from a comparison of our results with a harmonic oscillation. For prominence (1) the period was $82.2 \mathrm{~min}$ while that for prominence (2) was $76.7 \mathrm{~min}$.
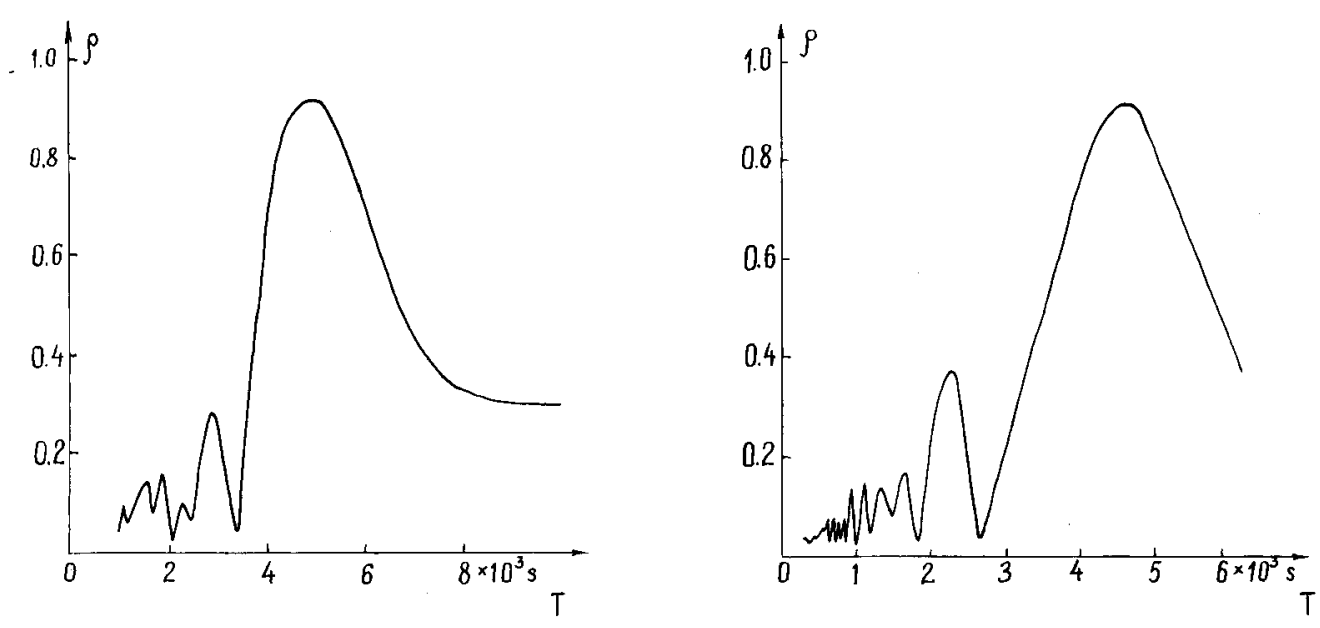

Fig. 2. Prominence correloperiodograms (1) and (2).

It is interesting that the prominence did not show any 5-min periods (or close to them) reported in literature for different prominence types.

In our opinion, it is now premature to given some interpretation of the observed oscillation periods in prominences. However detection of long-periodic oscillations in quiescent prominences is of great interest.

At the time of reduction and preparation of our observational data for publication we happened to know of a just published paper co-authored by Malville and Schindler (1981) who observed radial and torsional oscillations with a period about $75^{\mathrm{m}}$ in a loop prominence approximately $90 \mathrm{~min}$ before the onset of a limb flare. These oscillations have been interpreted in terms of a kink instability of a current carrying flux tube before 
the flare. It will be noted here that the prominences we observed were stable and not connected with flare phenomena. Therefore the above mentioned interpretation of the oscillations is not the only one possible.

We feel that further observations of the prominence velocity oscillations are needed at present.

\section{Acknowledgements}

We would like to thank Prof. V. E. Stepanov, Drs N. V. Klochek, G. V. Kuklin, M. N. Nikonova, Yu. M. Palachev, and V. G. Mikhalkovsky for their assistance with this work and helpful comments. We are also grateful to the anonymous referee for critical and helpful comments on our paper.

\section{References}

Kalinyak, A. A. and Vasilyeva, G. J.: 1971, Solar Phys. 16, 37.

Kobanov, N. I.: 1983, Solar Phys. 82, 237 (this volume).

Kopecký, M. and Kuklin, G. V.: 1971, Issledovaniya po geomagnetizmu, aeronomii i fizike Solntsa 2, 167.

Kotov, V. A., Severny, A. B., and Tsap, T. T.: 1978, Monthy Notices Roy. Astron. Soc. 183, 61.

Kotov, V. A., Severny, A. B., and Tsap, T. T.: 1982, Izv. Krymsk. Astrofiz. Obs. 65 (in press).

Lebedev, N. N., Grigoryev, V. M., Klochek, N. V., and Kobanov, N. I.: 1972, Certificate of authorship No. 335652 (U.S.S.R.). A technique for magnetic field strength measurements.

Malville, J. M. and Schindler, M.: 1981, Solar Phys. 70, 115. 